\title{
AN ALGORITHM FOR AUTOMATICALLY DETECTING DYSLEXIA ON THE FLY
}

\author{
Suraj Shrestha and Pietro Murano \\ Department of Computer Science, The Universal Design of ICT Research Group, \\ OsloMet - Oslo Metropolitan University, Postboks 4 St. Olavsplass 0130 Oslo, Norway
}

\begin{abstract}
There are different types of algorithms used in eye tracking technologies. These algorithms are divided into two main categories: feature-based and model-based. Feature-based technologies consist of threshold values, which are used to decide the presence or absence of features or determinant factors. While the model-based approach is an iterative search of a model parameter, which is the best fitting model that is a closest match to the image. However, these approaches have significant problems regarding computational speed and accuracy.

Similarly, there are different types of eye - tracking technologies, which depend on different types of technologies such as infrared video cameras and other technologies, which require specific calibration and setup and are quite expensive. Therefore, in this paper, we propose an alternative eye-tracking technology using a new eye-tracking algorithm, which is highly portable and independent of any hardware or software systems. In an evaluation the algorithm worked accurately for users with strong dyslexia. Participants had various positive and negative opinions regarding such an auto-detection system. Furthermore, we propose that such technology could be used to automatically modify the content of online material to better suit dyslexic users.
\end{abstract}

\section{KEYWORDS}

Automatic detection, dyslexia, evaluation, algorithm, interviews.

\section{INTRODUCTION}

The history of eye tracking studies is quite old. In 1879, a French ophthalmologist named Emile Java (1839 - 1907) described the movements of the eye during the text reading process. He observed the movements of the eyes during reading of the text using a mirror and stated that eye movements are not continuous, rather they are composed of many rapid movements called "saccades" along with stops called "fixations".

The "Photochronograph" was the first non-invasive and quite accurate eye-tracking device developed by Dodge and Cline in 1901 based upon corneal reflection [1]. However, the system only tracked the horizontal movements of the eye using a photographic plate [2]. Also, an improvement was made about four years later by an American psychologist named Charles $\mathrm{H}$. Judd, who developed a photo device that could track eye movements in both directions, i.e. horizontally and vertically [3].

DOI: $10.5121 /$ ijcsit.2018.10301 
Similarly, Edmund Huey developed an eye tracker device in 1908, using a small contact lens provided with a hole for the pupils and an aluminum pointer was connected to the lens in order to observe the gaze direction while reading [4].

Some studies such as the one by Miles Tinker, in 1930 tried to establish a relationship between typography and reading using eye-tracking technologies [5]. In 1947, Paul Fitts tried to establish a relationship between eye movements and the cognitive activity with the help of a video camera. He tried to record and study the ocular activities of airplane pilots while flying. His study suggested that the fixations were related to the importance of control and duration of fixations. These were related to how easy the information is interpreted [6]. The first head mounted eye tracker was developed by Hartridge and Thompson in 1948 [7].

In the 1970s, most of the research was focused towards finding relationships between human eye movements and perceptual/cognitive processes [2]. The entire eye - tracking research area took a great and unexpected turn in the 1980s, with the affordability and availability of personal computers. The researchers had a great instrument for data processing and they could now use it to find out further about eye tracking interactions between humans and computers. This opened huge opportunities for different user groups starting with people with disabilities, as these eye tracking technologies helped to create more accessible systems [8], [9]. Similarly, this new opportunity was also embraced by various business organizations, e.g. various marketing groups used these eye tracking technologies to understand further about the reading habits of people which could help to improve advertisements, announcements and product placements in various electronic media such as websites [10].

The research discussed in this paper, is related to an eye-tracking technology, which can help in the detection of dyslexia through the reading patterns. In this paper, the authors discuss the design and development of a prototype which can actively monitor or track the eye movements of a reader and based upon the above mentioned eye movements such as fixations, saccades, fixation durations and reading time the prototype can detect dyslexia and also automatically customize the textual content presentation to make the text more accessible for people with dyslexia.

In the first part of this paper, some relevant literature is discussed regarding dyslexia, eyetracking technologies and eye-movements etc. In the second part, the eye- tracking algorithm is discussed further in detail along with the main evaluative results. In the third part, the discussion, conclusions and suggestions for improvements are elaborated.

\section{RELEVANT LITERATURE ON EYE-TRACKING ALGORITHMS}

There are two particular types of eye -tracking algorithms: feature - based and model based[11]. Feature - based eye tracking technologies use the various features of the human eyes to detect distinctive features. They also consist of a threshold value, which is a decisive criterion for feature-based technologies to determine the presence or absence of a feature or determinant factor. While model-based approach is an iterative search of a model parameter which is a best fitting model which matches closest to the image. However, this approach can cause significant cost in terms of computational speed and accuracy [11]. There are various types of eye tracking technologies using infrared video cameras and other technologies, which require specific calibration and setup as well as being expensive. However, due to new webcam technologies, 
International Journal of Computer Science \& Information Technology (IJCSIT) Vol 10, No 3, June 2018

browser - based eye tracking technologies through simple and common webcams are gaining momentum with increased accuracy.

Starburst is an algorithm that is a combination of feature-based and model-based technologies. The main goal of this algorithm is to find out the location of the pupil center and the corneal reflection to relate vector differences between these measures and the coordinates in the scene images [11].

Similar to our eye tracking technology, there has been other research, which has used webcams for eye tracking. One of those studies was a study which used video images of mobile cameras to train neural networks [12]. Another study with self calibrating eye tracking technology which used a webcam consisted of pre-recorded gaze patterns [13]. Another eye tracking technology called PACE could self calibrate using the user interactions [14]. Unlike these previous technologies, our prototype uses a completely browser - based, self-calibrating and real-time technology which uses gaze-interaction relationships. Another important factor is that our prototype technology can be instantly included into any website for performing gaze tracking tasks.

Pupil detection is another aspect of the eye tracking technology. However, depending on pupil detection as a sole technique could cause failure, therefore, mapping from pixels to gaze location can be used [15]. However, "TurkerGaze" requires users to stare at calibration points while remaining motionless, which can be unrealistic for real time usage. Another eye movement feature, which plays an important role in the determination of specific eye movement patterns, is the detection of saccade. An algorithm called velocity-based saccade detection algorithm identifies the saccadic velocity peaks. A threshold value is determined such as $75 \%$ and any motion above the threshold value is considered as a saccade. However, there can be several other factors such as clear speed near the middle of the saccade [16] or the peak saccade velocity cannot be higher than a certain threshold [17]. This algorithm can thus help to distinguish between saccades and fixations. Many commercial software systems such as BeGaze 2.1 by SMI use this particular algorithm [18].

Another commercial software algorithm is 'Tobii fixation filter' developed by Olsson [19] which is used with low speed data using a double window technique [20].It uses two sliding windows on opposite sides of the current velocity sample and finds the average velocity within each window. The average values are subtracted to find out if the difference value can exceed a threshold, which can be detected as a saccade. The Hidden Markov Model (HMM) is another algorithm to classify data samples into saccades and fixations a using probabilistic model, which is based upon velocity information. The I-HMM model [21] uses two states, $S_{1}$ and $S_{2}$ which represent the velocity distribution of either fixation samples or saccade samples. I-HMM is found to be accurate and robust. However, it is very complex and requires many parameters. It needs eight parameters, which are estimated from similar sample data. Besides the two transition parameters for each state, it needs the observation probabilities in the form of velocity distributions. With the model parameters and the sequence of gaze positions, which are to be classified, the Viterbi algorithm can be used to map gaze points to states such as fixations or saccades. Due to the complexity of HMMs it is very difficult to use them in commercial applications, various parameters are required that must be set beforehand for use. 
International Journal of Computer Science \& Information Technology (IJCSIT) Vol 10, No 3, June 2018

\section{RELEVANT Literature ON EYE MOVEMENT STUDIES AND MEDICINE}

There have been several researches into auto-detection systems for various purposes including monitoring of patients with Mild Cognitive Impairment (MCI). One such research is by Hayes et al[22], which monitored elderly individuals in their homes over a period of time. One group had mild cognitive impairment (MCI) and the control group did not have MCI. Their aim was to discover if such monitoring of activities in the home could give an indicator regarding the onset or deterioration of MCI. They found that the technique of installing wireless sensors in the home was potentially unobtrusive and led to reliable results. This research is a useful proof of concept for our proposed auto-detection system since Hayes et al. [22] demonstrates that using technology to monitor individuals over time can give useful information about the onset or deterioration of MCI.

A similar concept was explored by Kaye et al. [23], where the authors looked at installing unobtrusive sensors in the homes of elderly patients with the aim of assessing aging etc.

Furthermore, in another study by Dwolatzky et al. [24] an evaluation of a computer based test system for detecting cognitive impairments was conducted. The authors concluded, based on empirical research, that the software was able to provide quite accurate results. However, the main issue with this kind of software is that it requires users to submit to a series of tests, where the results of these are aggregated to reach a conclusion about cognitive impairment of the user. This kind of testing, while useful in a 'medical' context, would not be useful or appropriate for interactive systems used in consumer-oriented technologies.

In a more recent body of work, researchers have begun to explore computerized assessment of cognitive and mental health related conditions. For example, Gutman, Moskovic, and Jeret[25], investigated the use of computerized testing of cognitive decline in persons with Down's syndrome and Alzheimer's disease. According to the authors, the test can be used to reliably quantify cognitive function over time. In a behavioral study of persons with depression, Saeb et al. [26] explored whether and to what extent mobile phone sensors could be used to detect behaviors linked to depressive symptoms. The authors argue that phone sensors offer the opportunity to monitor mental health conditions such as depression. In another study by Sikka et al. [27], the authors developed a prototype for automatically detecting and assessing pain in children. The authors argue that through "computer vision" and machine learning, software programs can assess and measure pain using facial recognition. Finally, Rello[28], investigated the use of computer based systems as a means for suggesting synonyms for difficult words. This was based on the knowledge that those with dyslexia will have an easier time reading a body of text that uses simpler words and other aids, e.g. certain fonts are known to be better for dyslexic readers etc. The authors tested a type of e-reader with some of these concepts allowing a user to select certain dyslexic-friendly options. However, this work did not examine the option of automatic detection of impairments and then subsequent automatic adaptation of the user's interaction.

There have also been efforts at detecting dyslexia automatically. One example is a US patent for automatic detection of dyslexia [29]. This used bespoke equipment to 'read' eye movements etc. However, while the ideas were good, the requirement of special equipment would not be suitable for an everyday type of interactive system, which would be potentially used by anyone. 
Therefore, our research uses research from medicine and rehabilitation science as a basis for examining the feasibility and prospective benefits of an auto-detection and-personalization system for removing barriers to using ICT for persons with cognitive disabilities. Our research focuses on the unobtrusive assessment of dyslexia leading to automatic detection and subsequent auto-adjustments are performed to create an easily accessible and personalized environment.

The use of eye tracking technologies to the fullest extent started with the study of human visual attention during the reading and information processing experiments [31]. There remains a long history behind the current eye tracking technologies, which dates back to the late 19th century. The first phase of eye tracking studies were conducted between 1879 and 1920 which cemented the foundation of eye movement through discovery of various eye movement patterns such as saccadic suppression, saccade latency, size of perceptual span etc.[31]. The second phase of the development of eye tracking technologies lies between 1930 and 1958 which was a more hands on research period combining the behaviorist movement in experimental psychology [31]. The third phase was between 1970 and 1998, which showed improvements in eye movement technologies in terms of accuracy and also became useful as diagnostic and interactive technologies. The diagnostic role consisted of gaining evidence of users' visual and attentional processes by recording eye movements over some stimulus [31]. With the advent of new and improved graphical displays, interactive patterns and stronger computational power, eye trackers are also now serving as a powerful means of interaction in an array of devices and applications.

There have been two major focus areas in terms of eye detection technologies: one is eye localization in the image and the other is the gaze estimation [32]. There are three major aspects of eye detection: the first is to find out the existence of eyes, the second is to determine the location and position of eyes using the center of the pupil or iris and the third is to track the eyes throughout the video images from frame to frame. Thus detected eyes are used to estimate and track the direct visual scope of a person or the line of sight.

\section{RELEVANT Literature ON EYE MOVEMENT STUDIES AND DYSLEXIA}

Almost all the eye movement studies are dependent on six major eye movement patterns. These six eye movements are measured in order to determine various conditions:

- Fixation: the time taken for processing image by Fovea.

- Saccade: the time taken by fovea to focus the attention from one image to another or the time interval between two fixations.

- Gaze Duration: It includes several fixations and also a relatively small amount of time for short saccades between fixations. Therefore, it is the cumulative duration and average spatial location of a series of consecutive fixations inside an area of interest [33].

- Area of interest: The area defined by a research team as per their needs such as area of a display device.

- Scan Path: Spatial arrangement of a sequence of fixations [33].

- Regressions are right to left movements or movements back to the previously read lines of text [34].

There have been several studies focusing on cognitive activities and eye movements [35]. These studies suggest that the reading patterns of people with and without dyslexia differ in various 
ways and can be inferred through their eye movement patterns [36], [35], [34]. In one study by Rayner [34], it is suggested that different aspects of eye movements such as shorter fixations are related to better ability to read whereas longer fixations infer problems with ability to read caused by overload of cognitive processing. Therefore, eye movement differs in dyslexic and nondyslexic individuals. Many studies have suggested that these eye movements are representative of reading disabilities. However, studies in[37], [38] and [39], [34] have shown that eye movements are related to reading disability and are reflections of underlying problems, which may be related to cognition.

In several non-reading tasks it has been shown that people with dyslexia have different patterns of eye movements than those without dyslexia [40], [30]. Research in[40] showed that the eye movement stability during fixation - was worse in children with dyslexia in comparison to those without dyslexia. However, replication studies have been inconclusive. In reading studies conducted by several researchers it is shown that readers with dyslexia tend to process less parafoveal information during each fixation in comparison to the other readers [41], [42].The visual field is categorized into three areas depending on the visual acuity limitations: fovea, parafovea and periphery. Parafoveal region is the $2-5^{\circ}$ range, which extends beyond the foveal region to about 15-20 characters. These studies, which include reading and non-reading tasks, suggest that there is an obvious difference in the eye movement patterns of dyslexic and nondyslexic people. There are different eye movement patterns such as saccades, fixation and regression. Saccades are the continuous eye movements made when reading or looking at objects. Within these saccades, our eyes have momentary fixation moments where they remain still for $200-300 \mathrm{~ms}$, which are called fixations. Regressions are right to left movements or movements back to the previously read lines of text [34].

Eye movement patterns of dyslexic readers tend to be different than those without dyslexia in terms of more frequency of fixations with longer durations, shorter saccades and more regressions [43], [44], [40], [45], [28]. The higher numbers of fixations are representative of dyslexia as people with dyslexia have relatively more fixations than people without dyslexia [46], [36]. Research on reading disabilities has employed eye-tracking technologies, and the data have been employed in various studies regarding reading and information processing tasks [34].

The literature reviewed in this section suggests that dyslexia is not just a reading disability but that reading disability represents broader and more complex physiological phenomena. In addition, the literature suggests that a cognitive disability may lead to a reduction in language processing capabilities, which is seen on the surface as a difficulty to read and understand text or language.

Our prototype consisted of two different aspects combined into one technology. The first part was the eye tracking technology and the second one was the automatic adjustment system. Our main objective was to understand the usage of eye tracking technology to distinguish between people with dyslexia from those without dyslexia. Besides this goal, we also needed to focus on some parameters to achieve this objective such as the application had to be simple in design, accessible for everyone, non-invasive in nature, secure and safe to use and the data that was collected must also be stored securely.

The prototype was based upon the findings from the previous studies, which suggested that the eye movement patterns of people with dyslexia were very different from those without dyslexia. 
International Journal of Computer Science \& Information Technology (IJCSIT) Vol 10, No 3, June 2018

These studies suggested that the reading patterns varied between dyslexic and non-dyslexic individuals.

The same was also true for non-reading tasks as well. These differences of eye movement patterns were displayed by various factors such as regressions, fixations durations, number of fixations and saccades. Although there were some studies conducted prior to our study regarding the effects of these factors on eye movement patterns of dyslexic readers, there was a research gap when it came to technologies, which could automatically detect these changes in patterns of eye movements.

\section{THE AlgORITHM AND PROTOTYPE}

Our prototype is a client-side eye tracking technology, which is completely written in JavaScript and uses various regression models, which match pupil positions, and various other eye features with respect to screen locations during a user interaction session. With the addition of very few lines of JavaScript code, our prototype can be integrated into any other website, software or secondary usage platform and our prototype will be activated to screen and detect dyslexia and provide customizations and suggestions according to the needs of the users.

It is simple in design and this simplicity makes it capable of running in real time in any JavaScript enabled browser. Unlike many other eye tracking solutions, it does not require a calibration model or preliminary calibration sessions, because it is enabled with an automatic learning capability which can learn and map new pupil positions, different eye features in correspondence to the screen coordinates. As mentioned above, our prototype is independent of hardware or software platforms. In terms of hardware, it uses a built-in webcam in any laptop or any other normal webcam to track the eyes. In terms of software, it is written in simple JavaScript, which can be easily used in almost all web platforms. It is easy to use as well as it has been proven to be quite reliable and accurate. The algorithm used for our prototype uses similar underlying factors as the prototype discussed in[47]. The algorithm uses a new method of storing and analyzing the eye tracking data. As a result of this process, a user can be distinguished as someone who has or has not dyslexia. Similarly, based upon this result, the appearance of textual contents is changed.

\subsection{Pupil Detection}

Our prototype uses three different facial and eye detection libraries namely clmtrackr[48], jsobject detect [49] and tracking.js [50]. It detects the eye regions and focuses on the pupil location. For accurate detection of pupil location, a comparative search of the entire regions is done to compare it for the highest contrast. This is done on the basis of the assumption that the iris is circular, darker and the pupil is located at its center.

\subsection{Mapping Pupil position on Screen}

To map the pupil on the screen, we use the $\mathrm{X}$ and $\mathrm{Y}$ coordinates. Here, we need to combine the $\mathrm{X}$ and $\mathrm{Y}$ coordinates to create a gaze point on the screen. Thus, we can obtain $n=(n 1 \ldots n N)$ pupil locations for which $X=(x 1 \ldots x N)$ and $Y=(y 1 \ldots y N)$, where $(n 1 \ldots n N)=(x 1+y 1) \ldots \ldots(x N+y N))$.

The $\mathrm{X}$ and $\mathrm{Y}$ coordinates, which are generated from the eye tracking data, are stored in the local storage. However, when it comes to local storage and JavaScript there are two particular limitations: the first one is that the size of the local storage is limited and the second one being 
that it is a temporary storage solution so data stored in local storage can be wiped out by another overlapping data storage operation.

In our case we needed a method to store and analyze our eye tracking data. JavaScript does not provide data storage facilities like other programming languages and therefore it was difficult for us to transmit the data from local storage into a permanent storage solution. Therefore, we have created our own storage solution to transmit the data from the local storage into a permanent storage solution, which in this particular case is a text file.

Therefore, we have developed a method, which can extract data from the local storage and then store it in a text file, which means the data can be easily viewed and analyzed further if required. This is a unique solution developed specifically for this particular application. Ridge regression model maps the eye pixels to gaze point locations [51]. As described above in section 4, there are various types of eye movements. Among them, the two major eye movements are saccades and visual fixations, which last for 200-500ms when the eyes focus on a specific area [34]. During this period of fixation, the information is retained for further cognitive processing. Therefore, this period of fixation is used in our ridge regression model.

A normal built-in webcam of a laptop was used for the purpose of user testing. The participants were shown five consecutive passages of Norwegian text on a simple web-type page. Each passage of text contained 77 words.

When the user first initiates the testing, an alert is generated, which informs the user that the webcam is going to be activated and their eye tracking data is going to be stored. Once the user agrees, the user is presented with the first passage and simultaneously the eye tracking data is continuously analyzed. After the user completes reading the passage and triggers an event to move on to the next passage, the eye tracking information from the first passage reading task is analyzed.

For every " $n$ " pupil location, there is a combination of $(\mathrm{x} 1+\mathrm{y} 1)$ coordinates. These coordinate values are stored in the local-storage. This " $n$ " pupil location can give us the number of fixations. If the number of fixations " $n$ " is found to be greater than the threshold value, and the reading time is found to be more than the respective threshold then the user is detected to be dyslexic. On the other hand, if the number of fixations and the reading time are found to be less than the threshold values, then the user is not detected as dyslexic. An alert will be generated at the end of the analysis, which will inform the user that he/she is dyslexic, or not. If the user is found to be dyslexic the user will be notified that the appearance of the textual content will be changed. Once the user agrees, the appearance of the textual content will be changed in terms of font-size, fonttype, line-spacing etc. If the user is not found to be dyslexic the appearance of the text will remain the same.

\subsection{Structure of the Prototype}

The prototype consists of three major parts:

1. Input and storage

2. Processing

3. Output 
International Journal of Computer Science \& Information Technology (IJCSIT) Vol 10, No 3, June 2018

1. Input and storage: - This part of the prototype is responsible for detecting the eyes and the various movement patterns within the eyes. The eye movement information in terms of number of fixations and the duration of reading time is stored.

2. Processing: The data stored was then analyzed. From various literature and previous experiments logic was derived for the prototype in terms of two particular logic statements for number of fixations and the reading time. In order to obtain better accuracy the two values were interlinked and if both conditions matched then the result would be positive otherwise it would be negative.

3. Output: Once the processing is completed, the output is shown which consists of two things. The first one is the detection of dyslexia, which can be positive or negative. The second thing is the automatic customization of textual content; if the user is found to have dyslexia then the appearance of the textual content changes otherwise there are no changes. The automatic customizations are set according to the standards set forth by various previous literature, research and dyslexia organizations. The structure of the prototype can be visualized in Figure 1 below and to further aid visualization and understanding, Figures 2 and 3 below show a use case diagram and integration flow diagram respectively.

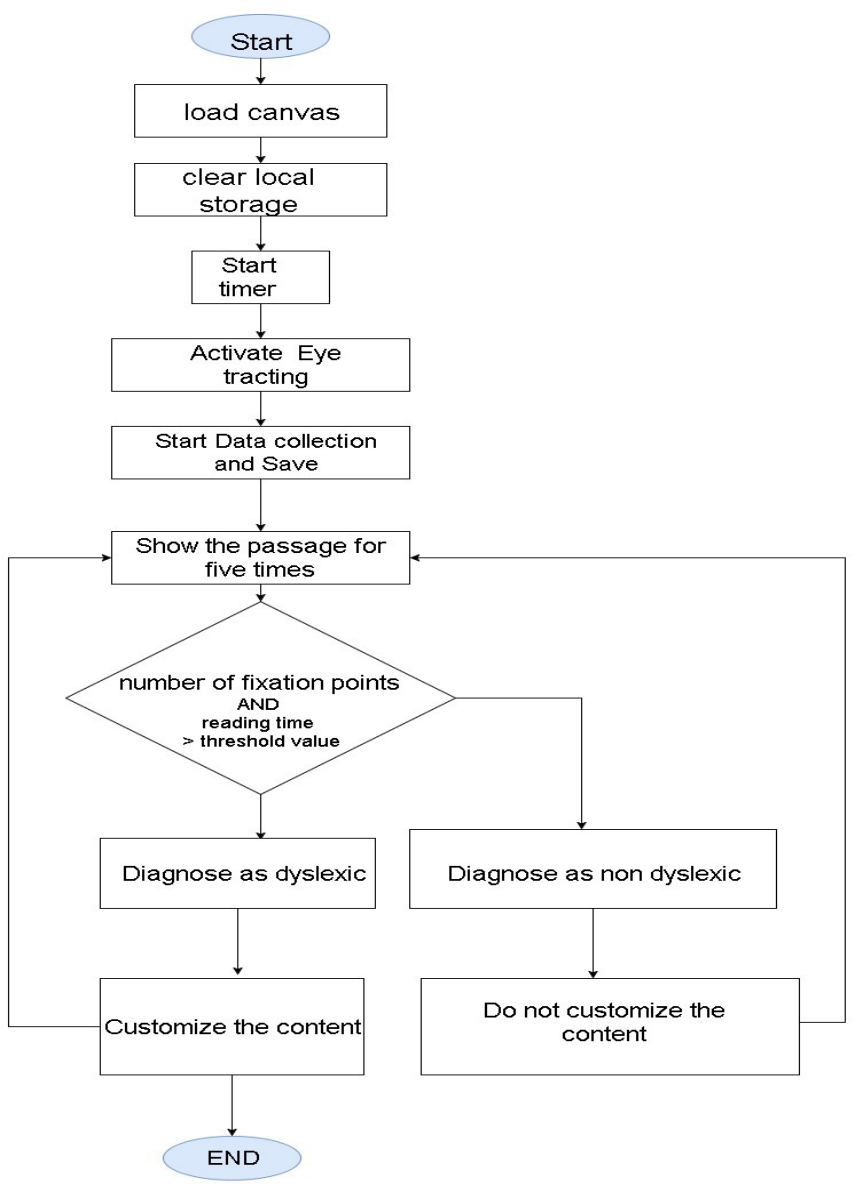

Fig. 1. Illustration of Prototype Structure 
International Journal of Computer Science \& Information Technology (IJCSIT) Vol 10, No 3, June 2018

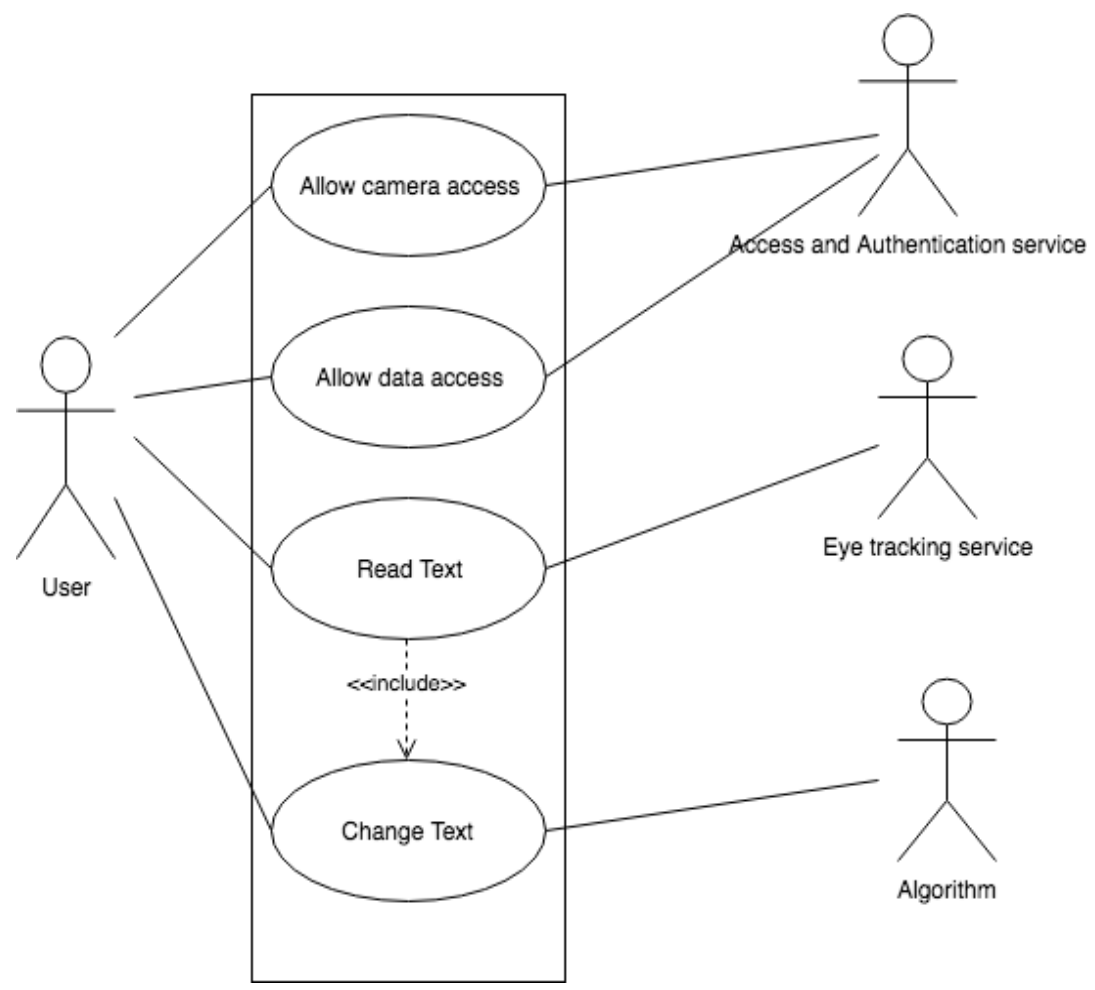

Fig. 2. Use Case Diagram

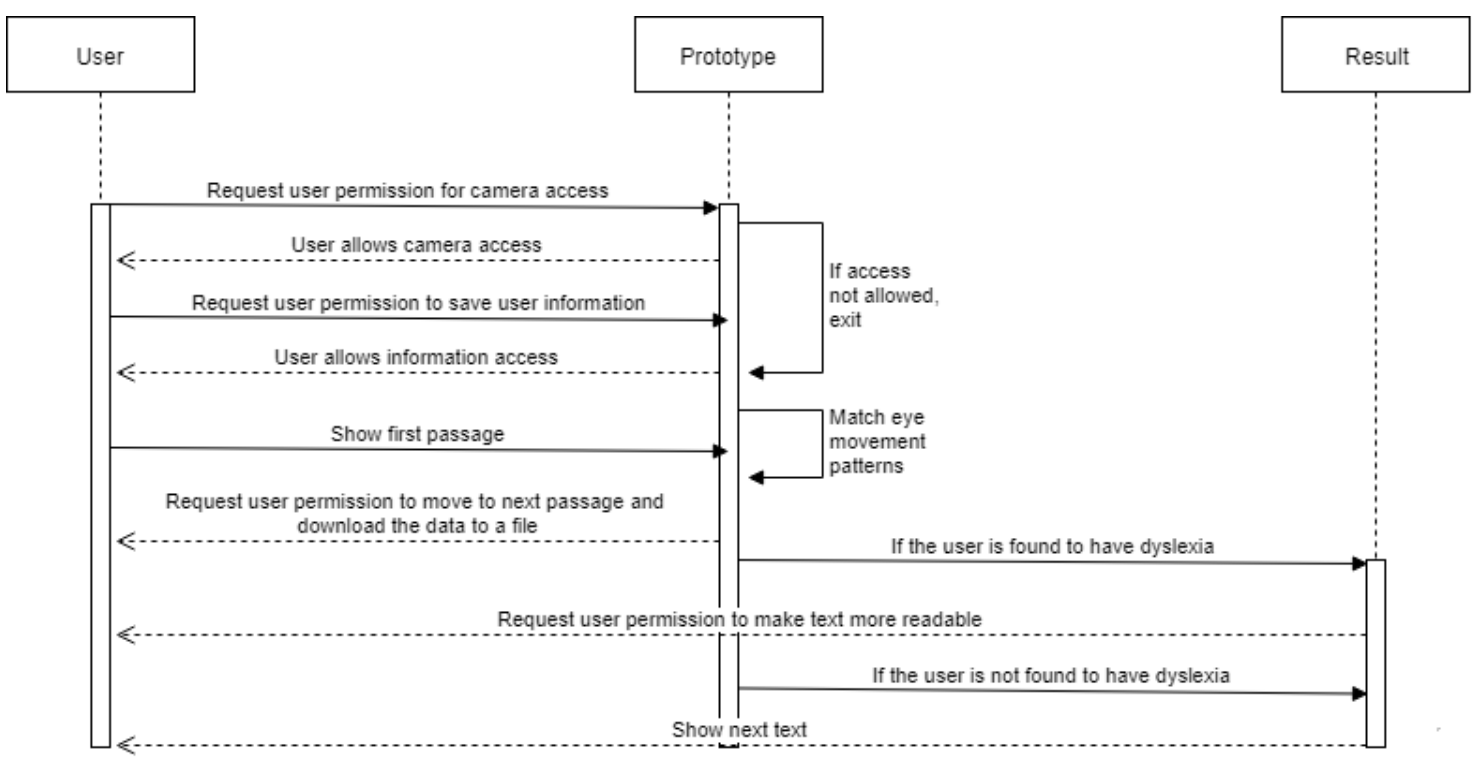

Fig. 3. Integration Flow Diagram 
International Journal of Computer Science \& Information Technology (IJCSIT) Vol 10, No 3, June 2018

\subsection{Testing and Evaluation}

Testing of the prototype was carried out qualitatively with a small sample of dyslexic users. This involved live testing of the prototype followed by a semi-structured interview. The interview process helped to gain further insights about various aspects of the prototype.

In carrying out user testing, the authors aimed to find out about the feasibility of such a system for possible real use and to begin discovering issues of possible usability of the prototype.

Four dyslexic participants who were all university students volunteered to participate in testing. They had different levels of dyslexia, which varied, from simple hindrances while reading to severe hindrances. All participants were Norwegian speakers. Although there are different forms of dyslexia, all of our participants were visual dyslexics. Ethical requirements in line with Norwegian guidelines were followed for this research [52].

From the user testing with the four participants, 3 out of 4 participants were found to be dyslexic. One participant was not found to be dyslexic since the participant had a mild form of dyslexia.

The semi-structured interviews centered around four basic questions:

1. What are the participants' experiences interacting with user interfaces?

2. How do the participants prefer to interact with user interfaces?

3. What are the participants' experiences using the prototype?

4. How do the participants expect to use the prototype in different settings?

A brief summary of the issues raised and discussed in the interviews is presented below.

For Question 1, several participants discussed the barriers that they experience in daily life and when using a variety of different technologies. According to one participant, their reading experience involved seeing text lines jumping and finding long text lines difficult to read. To help not lose their place in the text, they tended to use paper, their finger or mouse cursor. This avoided having to read the same line of text several times. Playing games online also posed difficulties because sometimes the text size and information varied.

Another participant discussed the barriers they experience when typing. If typing in English they had difficulty typing a word correctly enough so that the system could provide a suggestion. If the typing error was large the 'autocorrect' option will potentially give the wrong suggestion. The 'autocorrect' feature itself has accessibility issues for dyslexics, one important factor is that the suggestions provided are also in text form so it becomes difficult for dyslexics to understand the 'autocorrect' suggestions as well. In this case an alternative can be text that is more easily distinguishable or readable for dyslexics or a clear audio feedback which spells out the text suggestion for them.

The same participant also discussed how they experience watching television with subtitles. At times the subtitles were too fast, particularly if these were in English. This results in the subtitles being turned off.

The participant also went on to discuss their experiences using audio books. According to the participant audio books sometimes used bad quality audio. Mention was further made about a 
library for dyslexic users where audio books can be ordered. However, they have very bad sound quality and use a 'robotic' voice.

One participant discussed their reaction to encountering barriers. Whilst reading books, they felt frustrated by reading the same lines of text two or three times. As the eyes or focus cannot keep up then anger and distress can set in. This induces them to stop reading and have a break from reading.

Finally, the same participant discussed their experiences requesting accommodations from their school as a person with dyslexia. The participant felt that it would be good to have some 'technology' that helped them to read as one without dyslexia. They also did not want to have 'special' treatment as a dyslexic person. Their feeling was that it was not a problem in 'asking' for something to help them, but it was the 'receiving' because they are 'different'. This causes a barrier.

For Question 2, several participants also discussed how they prefer to interact with user interfaces. According to one participant, they felt that they could not use help manuals or read help manuals. They preferred to simply try 'something'. This participant also preferred reading white text on a black background and therefore will choose this option if available on a web site. They expressed the feeling that when 'dark mode' is available on a web site that it is at times difficult to find the option.

The same participant discussed how the design of certain user interface features affects their ability to focus. They felt that popup windows and anything on the user interface that 'moves' or distorts the focus are annoying. These are aspects that can make it easier to lose one's place in the text they are reading. They also discussed that popup windows asking for confirmations or advertisement popups can cause distractions. When they are reading text or working and suddenly a popup window appears then they can lose control of the document and sometimes they need to repeat reading the text as the pages get reloaded. This can lead to confusion, irritation and distraction.

It was also stated that having things simple can make things boring. However, if images with the text are included and options to link with other media are included these are good. It was felt that having images, icons or emojis make it easier to communicate. Many things that require one's attention are annoying, but different things to focus on are good.

Some participants discussed their preferences for personalizing user interfaces. Some felt that they wanted to keep control of the user interface. Some felt that personalization would be good for having books read and to provide better suggestions in 'autocorrect' aspects. They also discussed about the availability of a preference setting so that they could easily disable the autodetection and auto-adjustment feature in the event that they did not require it or found it to be difficult to use.

One of the participants reflected on their potential concerns of a personalized user interface and the benefits that personalization may have for themselves and others. They expressed negative opinions about a software application that recorded them or registered and stored information about them. The opinion was also expressed that changes to the user interfaces could be made depending on one's mood or feelings and that different modes could be saved for different 
situations. It was also felt that being able to easily change a dictionary for spelling and grammar would be helpful.

The participant went on to discuss that it would be beneficial if a user interface adapts to the user's needs rather than the user having to do it every time. However, they would also like to be informed about the changes prior to the changes taking place so that they can make informed decisions regarding whether such changes should take place for them or not.

For Question 3, the participants provided many insights on their experiences using the developed prototype. Some participants pointed out the challenges they experienced using the prototype. As the prototype is not a full application some small white spots on the screen are at times discernible by users whilst reading. These are in connection with the eye tracking done via the webcam. Some felt these took away one's focus from the text on the screen. Some felt that they could not go back to a previous page and read the content again.

Another participant similarly stated that the first paragraph was difficult to understand and took a long time to read due to having to re-read it. However it was deemed to be a clever thing to have a system that detects dyslexia automatically, even because many people are not tested for dyslexia.

Some participants also expressed some concerns over the prototype's ability to recognize characteristics of dyslexia correctly. Some pointed out that they want to maintain total control over the behavior of the prototype and that it is not good if the prototype makes changes without confirmation from the user. It was also stated that if the system changes the text in some way it would be good to have access to the original text too.

A different participant went on to reflect on several potential features for the prototype. One feature involved having clear descriptions of options in settings with descriptions of what they do. Things should be usable and understandable. There was also mention made that it would be good to have a feature allowing one to 'mark' words that one does not understand with the aim of better understanding the words by seeing, e.g. context, synonyms and perhaps being able to hear how the word is pronounced.

Another participant suggested a feature to divide up paragraphs that did not require users to activate it. One further suggestion was made regarding the navigation pattern of the text. Once the reader had moved to the end of the paragraph or the end of the page then the next page could be shown automatically.

For Question 4, the participants also discussed several applications for the prototype. Some participants discussed broadly how the prototype could be used in different settings. One participant reflected on aspects of the workplace, education, gaming and public sector websites and felt that it would not work well in these contexts. However, it was acknowledged that it may be useful in some circumstances, such as boring web sites, but would be annoying on web pages that were fine.

One participant pointed out that the prototype may be useful for younger people. The same participant reflected on more specific features and applications for the prototype, e.g. by making end-user licensing agreements understandable for someone who is not a lawyer. Concerning 
International Journal of Computer Science \& Information Technology (IJCSIT) Vol 10, No 3, June 2018

dyslexia detection, it would be good if the system could enlarge text where it happens to move quickly, e.g. in a 'chat' stream.

Another participant suggested a different context for online books as some content can be difficult to read. The participant had suggested that an application like this could be used as an added feature in some already available dedicated devices for reading books such as the Amazon Kindle.

Overall the detection process seems highly accurate when users have a strong form of dyslexia. However, the prototype seems less accurate with users who have a mild form of dyslexia.

The semi-structured interviews showed a mixed response regarding having such a tool. Some participants thought it a good idea, while some had reservations regarding certain aspects of such an auto-detection process. The authors believe that one of the reasons for some of the participants expressing some negative opinions was that it was perhaps not made clear enough to participants that the sample text would be different on an actual web site. It should have perhaps been made clearer that such a tool used in real life would be developed to be easily disabled if made into a web browser plug-in or add-on. Also, any privacy issues or issues of a user being 'labeled' as 'something' would be dealt with in a real life web browser setting. Certain participants perhaps did not fully understand (due to us perhaps not explaining well enough) that this prototype was merely testing the feasibility of such a solution.

\section{DISCUSSION AND CONCLUSION}

There are different medical tests, which are used to detect dyslexia. This process is both time and resource intensive since these tests take a long time. Therefore, many people remain undetected as dyslexics and they have to suffer from various reading disabilities throughout their lives. The Swedish Council on Technology Assessment in Health Care (SBU) has recently presented a systematic review of the scientific evidence for screening and diagnostic tests for children and adolescents with dyslexia [53]. According to this report, most of the tests that are used today lack scientific support. One of the key concerns raised in the report is that the existing tests are insufficiently evaluated with respect to their predictive validity, which leads to difficulties in concluding the usefulness of the tests that are in current practice [53].

Here, we have presented an eye-tracking algorithm, which can detect dyslexia and other similar reading disabilities. The two specific parameters regarding reading such as reading time and number of fixations are extracted and further processed. Each parameter is compared against their respective threshold value and if they are found to exceed the threshold value then the participant is considered to be dyslexic. For the purpose of increasing accuracy, both the parameters must have exceeded the threshold values in order to determine the participant to be dyslexic.

Our prototype is different from other eye-tracking technologies in terms of the innovative concept of detection of eye movements using a simple webcam located in a normal laptop. It is also independent of any hardware or software platforms. Most of the previous applications or eye detection technologies, which we came across in our research, were dependent on specific hardware platforms or specific software technologies. They also had further issues related to portability and interchange of data due to various data formats. The technologies, which have been developed for detection of dyslexia, are quite new and they still have a long way to go to 
become completely reliable and accurate as well. The data format used in our prototype is also highly interchangeable.

With the addition of some fairly simple code, the prototype can be added to any website and it will immediately start working because it is independent of any hardware or software systems. Another option is that the prototype could be developed into a plug-in or add-on for web browsers that is easily installed and works unobtrusively. Alternatively, browser manufacturers could incorporate this underlying technology as a core functionality of a web browser.

While we do believe that our prototype is a novel approach towards detection of dyslexia, it is important to state that dyslexia is a multi-faceted disability. There are various types of dyslexia, which vary in terms of phonological deficit, visual and spatial perceptions and cognitive impairments. Much prior research has debated over the source of dyslexia and its effects. However, in recent years, most of the research has concluded that dyslexia is a language-based disorder associated with a phonological deficit and it can affect the ability to read, process and comprehend written text [54]. With the support of these previous research findings, we make an assumption, that the language processing has a direct influence on the eye movements during reading. This assumption has been supported by various research in the fields of cognitive psychology and psycholinguistics [55]. Therefore, atypical eye movements are although a secondary consequence of dyslexia, eye tracking is an effective means for assessment of the processing demands that dyslexic readers experience while reading which creates a sound basis for developing predictive and automated models useful for screening [53]. Similarly, eye tracking during free viewing of natural images and videos have been implemented to differentiate subjects with Parkinson's disease, schizophrenia and autism spectrum disorders from control subjects [56], [57].

We believe that this is a novel approach towards detection of dyslexia and further improvements are necessary to refine the detection algorithm. In future, the algorithm can contain multiple parameters related to reading patterns as described above in section 4 . The at times perceivable spots on the user interface must also be removed. The user testing can be carried out with a higher number of participants to increase validity. Similarly, the user testing should also contain people with different types of dyslexia or other reading disabilities of a similar kind. Another approach can be a comparative study between two different types of participants; those who have dyslexia and those who have not.

This research shows that the two parameters of reading time and number of fixations can be used to detect dyslexia. However, further incorporation of several other parameters can increase accuracy. It has also shown that a simple webcam technology can be used for detection of dyslexia. It shows that eye tracking technologies can be made economically viable in the future.

\section{ACKNOWLEDGEMENTS}

The authors would like to acknowledge and thank the Norwegian Directorate for Children, Youth and Family Affairs (Barne, Ungdomsogfamiliedirektoratet - Bufdir) for the financial support given to this work. G. Anthony Giannoumis is acknowledged for his contribution to this project. 
International Journal of Computer Science \& Information Technology (IJCSIT) Vol 10, No 3, June 2018

\section{REFERENCES}

[1] Dodge, R., \& Cline, T. S. (1901). The angle velocity of eye movements. Psychological Review, 8(2), 145.

[2] Jacob, R. J., \&Karn, K. S. (2003). Eye tracking in human-computer interaction and usability research: Ready to deliver the promises. Mind, 2(3), 4.

[3] Shahzad, M. I., \&Mehmood, S. (2010). Control of articulated robot arm by eye tracking.

[4] Huey, E. B. (1908). The psychology and pedagogy of reading. The Macmillan Company.

[5] Tinker, M. A. (1963). Influence of simultaneous variation in size of type, width of line, and leading for newspaper type. Journal of Applied Psychology, 47(6), 380.

[6] Russell, M. C. (2005). Hotspots and hyperlinks: Using eye-tracking to supplement usability testing. Usability News, 7(2), 1-11.

[7] Hartridge, H., \& Thomson, L. C. (1948). Methods of investigating eye movements. The British journal of ophthalmology, 32(9), 581.

[8] Levin, S., Holzman, P. S., Rothenberg, S. J., \& Lipton, R. B. (1981). Saccadic eye movements in psychotic patients. Psychiatry Research, 5(1), 47-58.

[9] Hutchinson, T. E., White, K. P., Martin, W. N., Reichert, K. C., \& Frey, L. A. (1989). Humancomputer interaction using eye-gaze input. IEEE Transactions on systems, man, and cybernetics, 19(6), 1527-1534.

[10] Leggett, D. (2010, January 19). A Brief History of Eye-Tracking I UX Booth. Retrieved from http://www.uxbooth.com/articles/abrief-history-of-eye-tracking/

[11] Li, D., Winfield, D., \& Parkhurst, D. J. (2005). Starburst: A hybrid algorithm for video-based eye tracking combining feature-based and model-based approaches. Paper presented at the Computer Vision and Pattern Recognition-Workshops, 2005. CVPR Workshops. IEEE Computer Society Conference on.

[12] Baluja, S., \&Pomerleau, D. (1994). Non-intrusive gaze tracking using artificial neural networks

[13] Alnajar, F., Gevers, T., Valenti, R., \&Ghebreab, S. (2013). Calibration-free gaze estimation using human gaze patterns. Paper presented at the Proceedings of the IEEE International Conference on Computer Vision.

[14] Huang, M. X., Kwok, T. C., Ngai, G., Chan, S. C., \& Leong, H. V. (2016). Building a personalized, auto-calibrating eye tracker from user interactions. Paper presented at the Proceedings of the 2016 CHI Conference on Human Factors in Computing Systems.

[15] Xu, P., Ehinger, K. A., Zhang, Y., Finkelstein, A., Kulkarni, S. R., \& Xiao, J. (2015). Turkergaze: Crowdsourcing saliency with webcam based eye tracking. arXiv preprint arXiv:1504.06755.

[16] Smeets, J. B., \&Hooge, I. T. (2003). Nature of variability in saccades. Journal of neurophysiology, 90(1), 12-20.

[17] Nyström, M., \&Holmqvist, K. (2010). An adaptive algorithm for fixation, saccade, and glissade detection in eyetracking data. Behavior research methods, 42(1), 188-204

[18] Holmqvist, K., Nyström, M., Andersson, R., Dewhurst, R., Jarodzka, H., \& Van de Weijer, J. (2011). Eye tracking: A comprehensive guide to methods and measures: OUP Oxford

[19] Olsson, P. (2007). Real-time and offline filters for eye tracking

[20] Marple-Horvat, D. E., Gilbey, S. L., \& Hollands, M. A. (1996). A method for automatic identification of saccades from eye movement recordings. Journal of Neuroscience Methods, 67(2), 191-195

[21] Salvucci, D. D., \& Goldberg, J. H. (2000). Identifying fixations and saccades in eye-tracking protocols. Paper presented at the Proceedings of the 2000 symposium on Eye tracking research \& applications.

[22] Hayes, T. L., Abendroth, F., Adami, A., Pavel, M., Zitzelberger, T. A., \& Kaye, J. A. (2008). Unobtrusive assessment of activity patterns associated with mild cognitive impairment. Alzheimer's \& Dementia, 4(6), 395-405.

[23] Kaye, J. A., Maxwell, S. A., Mattek, N., Hayes, T. L., Dodge, H., Pavel, M., ... \&Zitzelberger, T. A. (2011). Intelligent systems for assessing aging changes: home-based, unobtrusive, and continuous assessment of aging. Journals of Gerontology Series B: Psychological Sciences and Social Sciences, 66(suppl_1), i180-i190. 
International Journal of Computer Science \& Information Technology (IJCSIT) Vol 10, No 3, June 2018

[24] Dwolatzky, T., Whitehead, V., Doniger, G. M., Simon, E. S., Schweiger, A., Jaffe, D., \&Chertkow, H. (2003). Validity of a novel computerized cognitive battery for mild cognitive impairment. BMC geriatrics, 3(1), 4.

[25] Gutman, M., Moskovic, E., \& Jeret, J. S. (2016). Computerised cognitive testing of individuals with Down's syndrome and Alzheimer's disease. Journal of Intellectual Disability Research, 60(2), 179181.

[26] Saeb, S., Zhang, M., Karr, C. J., Schueller, S. M., Corden, M. E., Kording, K. P., \& Mohr, D. C. (2015). Mobile phone sensor correlates of depressive symptom severity in daily-life behavior: an exploratory study. Journal of medical Internet research, 17(7).

[27] Sikka, K., Ahmed, A. A., Diaz, D., Goodwin, M. S., Craig, K. D., Bartlett, M. S., \& Huang, J. S. (2015). Automated assessment of children's postoperative pain using computer vision. Pediatrics, 136(1), e124-e131.

[28] Rello, L. (2014). DysWebxia: a text accessibility model for people with dyslexia

[29] Pavlidis, G. (1989). U.S. Patent No. 4,889,422. Washington, DC: U.S. Patent and Trademark Office.

[30] Pavlidis, G. T. (1978). The dyslexics erratic eye movements: Case studies. Dyslexia review, 1(1), 2228.

[31] Duchowski, A. T. (2002). A breadth-first survey of eye-tracking applications. Behavior Research Methods, Instruments, \& Computers, 34(4), 455-470.

[32] Hansen, D. W., \& Ji, Q. (2010). In the eye of the beholder: A survey of models for eyes and gaze. IEEE transactions on pattern analysis and machine intelligence, 32(3), 478-500.

[33] Lupu, R. G., Ungureanu, F., \&Siriteanu, V. (2013, November). Eye tracking mouse for human computer interaction. In E-Health and Bioengineering Conference (EHB), 2013 (pp. 1-4). IEEE.

[34] Rayner, K. (1998). Eye movements in reading and information processing: 20 years of research. Psychological bulletin, 124(3), 372.

[35] Hyönä, J., \& Olson, R. K. (1995). Eye fixation patterns among dyslexic and normal readers: effects of word length and word frequency. Journal of Experimental Psychology: Learning, Memory, and Cognition, 21(6), 1430

[36] Hutzler, F., \&Wimmer, H. (2004). Eye movements of dyslexic children when reading in a regular orthography. Brain and language, 89(1), 235-242

[37] Tinker, M. A. (1946). The study of eye movements in reading. Psychological bulletin, 43(2), 93

[38] Tinker, M. A. (1958). Recent studies of eye movements in reading. Psychological bulletin, 55(4), 215

[39] Rayner, K. (1985). The role of eye movements in learning to read and reading disability. Remedial and Special Education, 6(6), 53-60

[40] Eden, G., Stein, J., Wood, H., \& Wood, F. (1995). Differences in eye movements and reading problems in dyslexic and normal children. Ophthalmic Literature, 2(48), 137

[41] Rayner, K., Murphy, L. A., Henderson, J. M., \& Pollatsek, A. (1989). Selective attentional dyslexia. Cognitive Neuropsychology, 6(4), 357-378

[42] Underwood, N. R., \& Zola, D. (1986). The span of letter recognition of good and poor readers. Reading Research Quarterly, 6-19

[43] Adler-grinberg, D., \& Stark, L. (1978). Eye movements, scanpaths, and dyslexia. Optometry \& Vision Science, 55(8), 557-570

[44] Borsting, E. (2002). Dyslexia and Vision: LWW

[45] Lefton, L. A., Nagle, R. J., Johnson, G., \& Fisher, D. F. (1979). Eye movement dynamics of good and poor readers: Then and now. Journal of Reading Behavior, 11(4), 319-328

[46] De Luca, M., Di Pace, E., Judica, A., Spinelli, D., \&Zoccolotti, P. (1999). Eye movement patterns in linguistic and non-linguistic tasks in developmental surface dyslexia. Neuropsychologia, 37(12), 1407-1420

[47] Papoutsaki, A., Daskalova, N., Sangkloy, P., Huang, J., Laskey, J., \& Hays, J. (2016). WebGazer: scalable webcam eye tracking using user interactions.

[48] Mathias, A. (2015). clmtrackr: Javascript library for precise tracking of facial features via Constrained Local Models

[49] Garaizar, P., \&Guenaga, M. (2014). A multimodal learning analytics view of HTML5 APIs: technical benefits and privacy risks. Paper presented at the Proceedings of the Second International Conference on Technological Ecosystems for Enhancing Multiculturality 
International Journal of Computer Science \& Information Technology (IJCSIT) Vol 10, No 3, June 2018

[50] Lundgren, E., Rocha, T., Rocha, Z., Carvalho, P., \& Bello, M. (2015). tracking. js: A modern approach for Computer Vision on the web. Online]. Dosegljivo: https://trackingjs. com/[Dostopano 30. 5. 2016]

[51] Hoerl, A. E., \& Kennard, R. W. (1970). Ridge regression: Biased estimation for nonorthogonal problems. Technometrics, 12(1), 55-67

[52] Norwegian Centre for Research Data (2017) http://www.nsd.uib.no/nsd/english/index.html, Accessed December 2017

[53] Benfatto, M. N., Seimyr, G. Ö., Ygge, J., Pansell, T., Rydberg, A., \& Jacobson, C. (2016). Screening for Dyslexia Using Eye Tracking during Reading. PloS one, 11(12), e0165508

[54] Vellutino, F. R., Fletcher, J. M., Snowling, M. J., \& Scanlon, D. M. (2004). Specific reading disability (dyslexia): what have we learned in the past four decades? Journal of child psychology and psychiatry, 45(1), 2-40

[55] Rayner, K., Pollatsek, A., Ashby, J., \& Clifton Jr, C. (2012). Psychology of reading: Psychology Press

[56] Tseng, P.-H., Cameron, I. G., Pari, G., Reynolds, J. N., Munoz, D. P., \&Itti, L. (2013). Highthroughput classification of clinical populations from natural viewing eye movements. Journal of neurology, 260(1), 275-284

[57] Wang, S., Jiang, M., Duchesne, X. M., Laugeson, E. A., Kennedy, D. P., Adolphs, R., \& Zhao, Q. (2015). Atypical visual saliency in autism spectrum disorder quantified through model-based eye tracking. Neuron, 88(3), 604-616.

\section{AUTHORS}

Suraj Shrestha is a lecturer at the Oslo Metropolitan University. He has previously worked on a Bufdir research project. He has received his Master Degree in Universal Design of Information Communication Technology from Oslo Metropolitan University. His recent publications include Shrestha, S., \& Murano, P. (2016). A Universally Designed and Usable Data Visualization for A Mobile Application in the Context of Rheumatoid Arthritis. INTERNATIONAL JOURNAL OF ADVANCED COMPUTER SCIENCE AND APPLICATIONS (2016).His research interests include the universal design of ICT systems, accessible technologies, user interfaces, human computer interactions and artificial intelligence.

Dr Pietro Murano is a Computer Scientist and Associate Professor at OsloMet - Oslo Metropolitan University, Norway. Amongst other academic and professional qualifications he holds a $\mathrm{PhD}$ in Computer Science. His specific research areas are in Human Computer Interaction, Usability and Universal Design of software systems. He publishes regularly at refereed international levels and also supervises $\mathrm{PhD}$ level work. Further $\mathrm{Dr}$ Murano is a reviewer for various international conferences/journals. (www.pietromurano.org). 